\title{
Das östereichische Pensionssystem
}

Josef Wöss

\section{Einleitung}

„Ein starkes öffentliches Rentensystem ist möglich. Das Beispiel Österreich" - unter diesem Titel wurden in einer Publikation der Friedrich Ebert Stiftung die Ergebnisse einer 2016 erstellten Vergleichsstudie zu den Rentensystemen in Deutschland und Österreich zusammengefasst (Blank et al. 2016a, 2016b). Die Studie hatte enorme Unterschiede ans Licht gebracht.

Aufmerksamkeit erregte vor allem, dass die gesetzlichen Renten in Österreich viel höher sind als in Deutschland. Politiker, Experten und Medien interessieren sich seither für den österreichischen Weg. So waren z.B. der Sozialbeirat der Bundesregierung und Mitglieder des Bundestagsausschusses für Arbeit und Soziales auf Erkundungstour zur Rentenpolitik in Wien. Das politische wie auch das wissenschaftliche Interesse am österreichischen Modell ist auch auf die die sehr ähnliche Grundstruktur der Systeme zurückzuführen. Unterschiede bei Leistungshöhen, Versichertenkreis, Finanzierung, Mindestsicherung im Alter, etc. sind damit relativ einfach festzumachen.

In den wesentlich höheren Rentenzahlungen in Österreich kommt zum Ausdruck, dass dort - anders als in Deutschland - das Ziel der weitgehenden Lebensstandardsicherung im Alter durch die gesetzliche Rente nach wie vor Bestand hat, auch für die heute Jüngeren. Zentrale Schwachstelle des österreichischen Systems ist der hohe "gender pension gap“. Ebenso wie in Deutschland sind die Frauenrenten viel niedriger als die Renten der Männer, wenngleich auf deutlich höherem Niveau. Zur Gegensteuerung wurde bisher vor allem auf die Anrechnung von Kindererziehungszeiten und - vorgelagert zur Rente - auf eine Verbesserung der Erwerbschancen der Frauen gesetzt.

Im vorliegenden Beitrag wird im ersten Abschnitt ein Überblick über die zentralen Elemente des österreichischen Rentensystems gegeben, verbunden mit empirischen Daten und einem Ausblick in die Zukunft. Im zweiten Abschnitt geht es um den Vergleich zwischen Österreich und Deutschland. Anschließend wird die hohe Bedeutung einer positiven Ar- 
beitsmarktentwicklung für die Zukunft der Renten erörtert. Zuletzt wird in groben Zügen vorgestellt, welche Rentenpolitik von der Anfang 2020 angelobten neuen österreichischen Bundesregierung (Koalition ÖVP/ Grüne) zu erwarten ist.

\section{Alterssicherung in Österreich}

Eines der zentralen Charakteristika der Alterssicherung in Österreich ist die klare Schwerpunktsetzung bei den gesetzlichen Pensionen. ${ }^{1} 90 \%$ aller Pensionszahlungen stammen aus gesetzlichen Systemen, lediglich $4 \%$ aus betrieblicher und $6 \%$ aus privater Altersvorsorge. ${ }^{2}$ Auch für die heute Jüngeren setzt Österreich in hohem Maß auf gesetzliche Pensionen. Die Finanzierung der gesetzlichen Systeme erfolgt im Umlageverfahren. Die Alterssicherung ist damit in jeder Generation eng mit der wirtschaftlichen Situation des Landes und insbesondere mit dem Arbeitsmarkt - der Zahl der Beitragszahler und der Höhe der Beitragsgrundlagen - verknüpft.

Zentrales Ziel des gesetzlichen Systems ist „die Erhaltung eines angemessenen Lebensstandards und der damit verbundenen Möglichkeiten sozialer, wirtschaftlicher, politischer und kultureller Teilhabe." ${ }^{3}$

\subsection{Reformen}

Seit den 1980er-Jahren sind die Pensionen eines der zentralen sozialpolitischen Reformthemen. Sowohl in der gesetzlichen Pensionsversicherung als auch in den Beamtenversorgungssystemen wurde das Leistungsrecht in mehreren Etappen umfassend umgestaltet. Primäre Ziele waren die Eindämmung des tendenziellen Kostenanstiegs und - damit verbunden - die Anpassung an den demografischen Wandel. Ein wesentliches Element war dabei immer wieder die Anhebung des lange Zeit sehr niedrigen, effektiven Pensionsantrittsalters. In Teilbereichen gab es auch Leistungsverbesse-

1 In Österreich werden Rentenzahlungen aus öffentlichen Systemen generell als Pensionen bezeichnet. Das Gegenstück zur deutschen Rentenversicherung heißt in Österreich Pensionsversicherung.

2 Url (2012).

3 Bundesministerium für Arbeit, Soziales, Gesundheit und Konsumentenschutz (2018), S. 168. 
rungen, insbesondere bei der Anrechnung von Kindererziehungszeiten und zuletzt auch bei der Mindestsicherung und bei den Regelungen für Langzeitversicherte.

Besonders umstritten war die Pensionsreform 2003, die von der damaligen Koalition von Österreichischer Volkspartei (ÖVP) und Freiheitlicher Partei Österreichs (FPÖ) unmittelbar nach Neubildung der Regierung ${ }^{4}$ mit der Vorlage eines Gesetzesentwurfes eingeleitet wurde. Vorgesehen war eine massive Kürzung der Leistungen aus der gesetzlichen Pensionsversicherung, inklusive Abschaffung aller vorzeitigen Alterspensionen. Im Gegenzug wurden - ähnlich wie in den deutschen Rentenreformen zu Beginn der 2000er-Jahre - ein Ausbau der Betriebspensionen und eine verstärkte öffentliche Förderung der privaten Altersvorsorge in Aussicht gestellt.

Die geplanten radikalen Änderungen scheiterten an einer breiten Ablehnungsfront, die u.a. in landesweiten Warnstreiks der Gewerkschaften Ausdruck fand. Der Protest richtete sich in erster Linie gegen die geplanten massiven Verschlechterungen im gesetzlichen System, damit verbunden aber auch gegen die angekündigte Umstellung auf ein „Drei-SäulenModell“ im Sinne der Weltbankvorschläge aus den 1990er Jahren. Dem Regierungsvorhaben wurde entgegengehalten, dass der massive Ausbau kapitalbasierter Zusatzpensionen keine Kosteneinsparung, sondern bestenfalls eine Kostenverschiebung - und in der Aufbauphase sogar massive Mehrkosten - mit sich bringen würde. Verwiesen wurde auch auf die Gefahr einer Fragmentierung der Alterssicherung je nach Finanzkraft der Unternehmen bzw. der Individuen und auf die Risiken einer Anbindung an die internationalen Finanzmärkte.

Die Regierung musste letztlich - unter Druck auch aus den eigenen Reihen - massive Abstriche gegenüber ihrem ursprünglichen Konzept machen, insbesondere bei der Ausgestaltung des ein Jahr später beschlossenen neuen „Pensionskonto-Rechts“ für ab 2005 neu erworbene Pensionsanwartschaften. In mehreren zentralen Punkten wurde den Forderungen des Österreichischen Gewerkschaftsbundes (ÖGB) Rechnung getragen, wie

4 Die erste ÖVP-FPÖ Koalitionsregierung wurde im Jahr 2000 gebildet. Im Herbst 2002 kam es wegen internen Zerwürfnissen in der FPÖ zum Rücktritt mehrerer FPÖ-Minister. Kanzler Schüssel (ÖVP) entschied sich für vorgezogene Neuwahlen. Großer Gewinner der Wahl war die ÖVP, großer Verlierer die FPÖ. Anders als von vielen erwartet kam es in Folge zu einer Fortsetzung der Regierungszusammenarbeit der beiden Parteien. 
z.B. bei der Beibehaltung eines leistungsdefinierten Systems ${ }^{5}$ auch für die Jüngeren und bei der - im Vergleich zur früheren Rechtslage - viel besseren Aufwertung zurückliegender Entgelte bzw. bereits erworbener Pensionsanwartschaften. Damit wurden die leistungseinschränkenden Effekte der aus dem ursprünglichen ÖVP-FPÖ-Konzept übernommenen langfristigen Umstellung der Pensionsberechnung von der früheren Bezugnahme auf „die besten 15 Jahre“ auf „Lebensdurchrechnung“ erheblich abgeschwächt.

Ein weiterer - vor allem für die Transparenz des Systems - sehr wichtiger Reformschritt erfolgte 2013 mit dem Beschluss, alle vor dem 1.1.2014 erworbenen Pensionsanwartschaften zu diesem Stichtag in einen Leistungsanspruch umzurechnen und diesen in Form einer "Erstgutschrift“ auf den bereits 2005 geschaffenen individuellen Pensionskonten auszuweisen.

In den letzten Jahren waren die gesetzlichen Änderungen auf die $\mathrm{Zu}$ gangsregeln zu den gesundheitsbedingten Frühpensionen fokussiert, vor allem durch die Streichung der Zuerkennung befristeter Invaliditätspensionen und deren Ersatz durch Rehabilitationsgeld und -maßnahmen. Erschwert wurde auch der Zugang zu „vorzeitigen“ Alterspensionen, z.B. wurde bei der „Korridorpension“ die Zahl der erforderlichen Versicherungsjahre von 37,5 auf 40 Jahre angehoben.

Die letzte wesentliche Rechtsänderung wurde im Herbst 2019 beschlossen. Anknüpfend an eine früher geltende Regelung, werden bei Langzeitversicherten auch bei Pensionsantritt vor dem Regelpensionsalter keine Abschläge mehr verrechnet (siehe unten, Fußnote 14).

\subsection{Kreis der Versicherten}

Anders als in der deutschen Rentenversicherung sind in Österreich alle Erwerbstätigen einschließlich der Selbständigen pflichtversichert, wobei die Pensionen der Selbständigen in einem eigenen Versicherungsträger verwaltet werden. Gesetzliche Versorgungssysteme außerhalb der Pensions-

5 Die Regierung wollte eine Umstellung auf ein beitragsdefiniertes System. Die Pensionshöhe sollte nach diesem Konzept durch Umrechnung der bis zum Pensionierungszeitpunkt eingezahlten Beiträge in einen Pensionsanspruch - unter Berücksichtigung der durchschnittlichen Restlebenserwartung - ermittelt werden. Im Hintergrund stand die Absicht, langfristig auf die Zuzahlung eines „Bundesbeitrags“" (siehe 1.6.) verzichten zu können. Das Leistungsniveau der gesetzlichen Pensionen wäre damit ganz erheblich nach unten gegangen. 
versicherung gibt es im Wesentlichen nur für die Beamten. Allerdings erfolgt auch dort in mittel- bis langfristiger Perspektive eine Angleichung des Beitrags- und Leistungsrechts an die Regelungen in der gesetzlichen Pensionsversicherung. ${ }^{6}$ Nur geringfügig Beschäftigte ${ }^{7}$ und von den freien Berufen die Rechtsanwälte sind nicht obligatorisch in gesetzliche Systeme eingebunden.

Aktuell (2018) sind - bei einer Gesamtbevölkerung von 8,8 Mio. 3,47 Mio. Unselbständige und 589.000 Selbständige in der gesetzlichen Pensionsversicherung versichert. In den letzten 10 Jahren ist die Zahl der Versicherungsverhältnisse bei den Unselbständigen um 16\% und bei den Selbständigen um $15 \%$ gestiegen (Hauptverband 2019a).

Das Leistungsspektrum der gesetzlichen Pensionsversicherung umfasst neben den Alterspensionen auch Invaliditäts- und Hinterbliebenenpensionen. Darüber hinaus werden z.B. auch Präventions- und Rehabilitationsleistungen geboten.

\subsection{Pensionsalter / Pensionsarten}

Das gesetzliche Regelpensionsalter ist 65. Für Frauen gilt aktuell noch ein Übergangsrecht mit der Regelaltersgrenze $60 .{ }^{8}$ Anders als in vielen anderen Ländern wurde bisher keine Anhebung des Regelpensionsalters über das 65. Lebensjahr hinaus beschlossen. Forderungen in diese Richtung z.B. von der EU-Kommission und von den österreichischen Arbeitgeberverbänden - sind am Widerstand vor allem des ÖGB gescheitert. Auch die von 2017 bis 2019 amtierende ÖVP-FPÖ-Regierung hat sich gegenüber der EU-Kommission klar gegen eine Anhebung des Regelpensionsalters positioniert: „Eine Koppelung des Pensionsantrittsalters an die Lebenserwartung oder eine Erhöhung des gesetzlichen Pensionsantrittsalters, wie von der Europäischen Kommission im Länderbericht bzw. in den länderspezi-

6 Auf Bundesebene gilt volle Angleichung bei Verbeamtung ab 2005, bei früherem Eintritt in den Beamtenstatus kommt ein Übergangsrecht zum Tragen.

7 Geringfügig Beschäftigte haben die Möglichkeit zu einer begünstigten Selbstversicherung in der Gesetzlichen Pensions- und Krankenversicherung. Zu beachten ist, dass bei mehreren parallelen Arbeitseinkommen immer auf das Gesamteinkommen abgestellt wird - liegt dieses über der Geringfügigkeitsgrenze von € 460,66 (2020), kommt die Pflichtversicherung zum Tragen.

8 Geburtsjahrgänge vor 6/1964 können bereits mit 60 abschlagsfrei in Pension gehen, bei späterem Pensionsantritt erhalten sie Zuschläge. Für Geburtsjahrgänge ab 6/1968 ist das Regelpensionsalter wie bei den Männern 65. Für die Geburtsjahrgänge dazwischen erfolgt eine schrittweise Anhebung. 
fischen Empfehlungen nahegelegt, wird von der Bundesregierung nicht in Erwägung gezogen“ (Nationales Reformprogramm; Bundeskanzleramt 2019).

Als Alternative zur Anhebung der gesetzlichen Altersgrenzen wird auf eine nähere Heranführung des im internationalen Vergleich niedrigen effektiven Pensionsantrittsalters an das Regelpensionsalter 65 gesetzt. 2018 lag das effektive Pensionsantrittsalter bei den Männern im Durchschnitt bei 61,3 und bei den Frauen bei 59,3 Jahren (Alters- und Invaliditätspensionen). Die Tatsache, dass hier erhebliche Änderungen im Gange sind, zeigt der Blick auf die Entwicklung der letzten Jahre: Seit 2010 wurde bei den Männern ein Anstieg um 2,4 und bei den Frauen ein Anstieg um 2,3 Jahre verzeichnet (Hauptverband 2011, 2019b). ${ }^{9}$

Es gibt drei Formen der Alterspension: ${ }^{10}$

- Regelalterspension (ab 65; Voraussetzung: 15 Versicherungsjahre ${ }^{11}$ )

- Korridorpension (ab 62; Voraussetzung: 40 Versicherungsjahre)

- Schwerarbeitspension (ab 60; Voraussetzung: 45 Versicherungsjahre und 10 Jahre anerkannte Schwerarbeit innerhalb der letzten 20 Jahre vor Pensionsantritt)

\subsection{Pensionshöhe}

Als Leistungsziel der gesetzlichen Pensionsversicherung gilt die Formel 80/45/65, d.h.

- $80 \%$ (Brutto-)Ersatzrate bezogen auf das Lebensdurchschnittseinkommen bei

- Vorliegen von 45 Versicherungsjahren und

- Pensionsantritt zum Regelpensionsalter 65.

Grundlage ist eine jährliche Pensionsgutschrift in Höhe von 1,78\% des versicherten Jahreseinkommens $(1,78$ x $45=80,1)$. Rentengutschriften gibt es z.B. auch für Zeiten der Arbeitslosigkeit und für Zeiten der Kindererzie-

9 Näheres zur Entwicklung des effektiven Pensionsantrittsalters siehe Türk (2019).

10 Als weitere Form der Alterspension könnte die Langzeitversichertenregelung betrachtet werden (siehe unten, Fußnote 14). Auf die gesundheitsbedingten Invaliditätspensionen und auf Hinterbliebenenpensionen kann im Rahmen dieses Beitrags nicht eingegangen werden.

11 Von diesen 15 Versicherungsjahren müssen mindestens sieben Jahre auf Grund einer Erwerbstätigkeit erworben sein. 
hung. Als Kindererziehungszeit werden pro Kind vier Jahre ${ }^{12}$ angerechnet, auf Basis einer jährlich valorisierten Beitragsgrundlage in Höhe von $€ 1.922,59$ (2020). ${ }^{13}$ Die daraus resultierenden monatlichen Pensionsgutschriften erfolgen additiv zu Gutschriften aus einem versicherten Erwerbseinkommen, das innerhalb dieser vier Jahre erzielt wird. Die Summe der bereits erworbenen Anwartschaften und die jährliche Gutschrift werden auf dem individuellen Pensionskonto ausgewiesen. Bis zum Pensionsantritt erfolgt eine jährliche Anpassung des Pensionsguthabens entsprechend der durchschnittlichen Entwicklung der Löhne bzw. der Beitragsgrundlagen. Tabelle 1 zeigt die durchschnittliche Höhe der 2018 neu zu erkannten Alterspensionen.

Bei Inanspruchnahme einer Korridorrente vor Erreichung des 65.Lebensjahres gibt es pro Jahr einen Abschlag in Höhe von 5,1\%. Liegen 45 Pflichtversicherungsjahre vor, wird seit 1.1.2020 kein Abschlag mehr verrechnet. ${ }^{14}$ Erfolgt der Rentenantritt nach 65, so gibt es pro Jahr (für maximal 3 Jahre) einen Zuschlag von 4,2\%. Bei der Schwerarbeitspension kommt ein begünstigter Abschlag von 1,8\% pro Jahr zum Tragen, liegen 45 Pflichtversicherungsjahre vor, fällt der Abschlag weg. ${ }^{15}$

Die Pensionszahlungen sind voll (lohn-)steuerpflichtig. Vorgelagert wird von der Bruttopension der Beitrag zur Krankenversicherung (5,1\%) abgezogen.

12 Ist der Altersabstand zwischen Kindern weniger als 4 Jahre, werden überschneidende Zeiten nur einmal berücksichtigt.

13 Der Betrag orientiert sich am Medianeinkommen der Frauen (ohne Berücksichtigung von Sonderzahlungen).

14 Im September 2019 wurde - mit Wirkung für Pensionsantritte ab 1.1.2020 - ein Gesetz beschlossen, das bei Langzeitversicherten mit zumindest 45 Pflichtversicherungsjahren aus Erwerbstätigkeit (anrechenbar sind lediglich Kindererziehungszeiten im Ausmaß von maximal 5 Jahren) den Wegfall aller Abschläge vorsieht. Der Gesetzesbeschluss knüpft - in modifizierter Form - an eine 2014 abgeschaffte Sonderregelung für Langzeitversicherte an. Unklar ist, ob bzw. wie lange das neue Recht Bestand haben wird. Die Anfang 2020 neu gebildete Regierung (Koalition ÖVP-Grüne) hat eine Änderung angekündigt.

15 Siehe Fußnote 14. 
Tabelle 1: Durchschnittliche Höhe der 2018 neu zuerkannten Alterspensionen" (Unselbständige / Jahreszwölftel*:)

\begin{tabular}{lcccc}
\hline & \multicolumn{2}{c}{ Männer } & \multicolumn{2}{c}{ Frauen } \\
& brutto & netto & brutto & netto \\
\hline Angestellte & $€ 3.094$ & $€ 2.414$ & $€ 1.772$ & $€ 1.573$ \\
Arbeiter & $€ 2.224$ & $€ 1.857$ & $€ 1.091$ & $€ 1.035$ \\
\hline
\end{tabular}

Quelle: Hauptverband (2019c); Netto-Beträge: eigene Berechnungen (Finanzministerium Brutto-Netto-Rechner)

Anmerkungen:

" arithmetisches Mittel; ohne zwischenstaatliche Teilleistungen;

* In Österreich gibt es sowohl bei den Löhnen als auch bei den Pensionen 14 Monatszahlungen pro Jahr. Zur leichteren Vergleichbarkeit mit Deutschland werden in der Tabelle die Jahreszwölftel ausgewiesen (bei den Nettowerten ist die begünstigte Besteuerung der 13. und 14. Monatspension aliquot berücksichtigt).

Für die laufenden Pensionen sieht das Pensionsrecht eine jährliche Anpassung nur mit der Inflationsrate vor (die Regelung gilt auch für die Beamtenpensionen). Häufig wird die konkrete Anpassung allerdings in Sondergesetzen geregelt, in denen z.B. festgelegt wird, dass niedrige Pensionen mit einem höheren Wert angepasst werden. So wurde z.B. für die Anpassung zu Jahresbeginn 2018 - ausgehend von einer Inflationsrate von 1,6\% - mittels Sondergesetz folgende Regelung getroffen: Pensionen bis $€ 1.500$ wurden mit 2,2 \% angepasst. Für höhere Pensionen im Bereich bis € 3.355 wurde der Anpassungssatz schrittweise auf 1,6\% und darüber hinaus - bis $€ 4.980$ - bis auf null reduziert. ${ }^{16}$

Die grundsätzliche Anpassung nur mit der Inflationsrate trägt - neben Struktureffekten - mit dazu bei, dass die Bestandspensionen aus der Pensionsversicherung im Durchschnitt um etwa $10 \%$ niedriger sind als die Neuzugangspensionen. Bei männlichen Angestellten liegt dieser Wert bei $€ 2.406$ brutto, bei weiblichen Angestellten bei $€ 1.394$. Bei Arbeitern ist die durchschnittliche Höhe der Bestandspensionen $€ 1.690$ bei Männern bzw. $€ 856$ bei Frauen (Werte 2018 / Hauptverband 2019d).

16 Die Anpassungen unterhalb der Inflationsrate betrafen de facto nur hohe Beamtenpensionen. 
Die ausgewiesenen Werte machen deutlich:

a) Bei höheren Pensionen gibt es durch das Wirksamwerden der Steuerprogression eine erhebliche Differenz zwischen Brutto- und Nettopension (siehe Tab 1).

b) Die Pensionen der Frauen sind im Durchschnitt wesentlich niedriger als die Männerpensionen.

Die hohen geschlechtsspezifischen Unterschiede liegen in den sehr unterschiedlichen Erwerbsverläufen begründet. Viele Frauen haben lange Erwerbsunterbrechungen oder lange Teilzeitphasen und/oder arbeiten in schlecht entlohnten Branchen bzw. generell in schlechterer Entlohnung.

\subsection{Mindestsicherung im Alter}

Pensionen, die unter einem bestimmten Richtsatz liegen, werden - wenn kein entsprechendes Eigen- oder Partnereinkommen vorliegt - mit der sogenannten „Ausgleichszulage“ auf diesen Richtsatz aufgestockt. In mehreren Reformschritten der letzten Jahre wurde das vorher einheitliche System der Richtsätze auf ein abgestuftes System umgestellt: Bei Vorliegen einer bestimmten Zahl an Erwerbsjahren wird nun durch Bonuszahlungen auf höhere Werte aufgestockt.

Tabelle 2: Ausgleichszulagen-Richtsätze / brutto (2020)

Alleinstehende

$€ 966,65$

Aufstockung („Bonus“) für Personen mit

30 Erwerbsjahren auf

$€ 1.080,00$

Aufstockung („Bonus“) für Personen mit

40 Erwerbsjahren auf

Ehepaare

$€ 1.524,99$

Aufstockung („Bonus“) für Personen mit

40 Erwerbsjahren auf

$€ 1.782,00$

Eigene Darstellung.

Ebenso wie die Pensionen werden die Ausgleichszulagen 14mal pro Jahr ausbezahlt. Finanziert werden die Ausgleichszulagen zu $100 \%$ aus Bundesmitteln. Die Administration erfolgt durch die gesetzliche Pensionsversicherung. 
Voraussetzung für den Bezug einer Ausgleichszulage ist das Vorliegen eines Pensionsanspruchs. Wer die dafür erforderlichen Versicherungsjahre nicht erreicht, ist bei Armut im Alter auf Leistungen aus der von den Sozialämtern administrierten Sozialhilfe angewiesen (mit niedrigeren Leistungen, Vermögensprüfung, etc.).

\subsection{Finanzierung}

Die Finanzierung der Leistungen der gesetzlichen Pensionsversicherung erfolgt in erster Linie durch die laufenden Beitragszahlungen, ergänzend gibt es den „Bundesbeitrag“. Der Beitragssatz liegt bei den Unselbständigen seit 1988 unverändert bei 22,8\%. Davon entfallen 12,55\% auf den Arbeitgeber und 10,25\% auf den Arbeitnehmer. Die Beitragsbemessungsgrenze („Höchstbeitragsgrundlage“) liegt bei $€ 5.370$ pro Monat bzw. $€ 75.180$ pro Jahr (5.370 x 14; Werte 2020). ${ }^{17}$ Der von den Selbständigen (Gewerbetreibende) zu entrichtende Eigenbeitrag beträgt 18,5\%. Die Differenz zum Gesamtbeitrag von 22,8\% wird aus Bundesmitteln beglichen.

Tabelle 3: Entwicklung des Bundesbeitrags zur gesetzlichen Pensionsversicherung 2005-2018 (Unselbständige + Selbständige)

\begin{tabular}{ccc}
\hline & $\begin{array}{c}\text { Bundesbeitrag } \\
\text { in des Pensionsaufwands }\end{array}$ & $\begin{array}{c}\text { Bundesbeitrag } \\
\text { in \% des BIP }\end{array}$ \\
\hline 2005 & $18,7 \%$ & $1,8 \%$ \\
2010 & $22,8 \%$ & $2,2 \%$ \\
2015 & $21,6 \%$ & $2,2 \%$ \\
2018 & $17,9 \%$ & $1,8 \%$ \\
\hline
\end{tabular}

Quelle: Hauptverband der österreichischen Sozialversicherungsträger, Handbuch der österreichischen Sozialversicherung, diverse Jahrgänge

Ein weiteres zentrales Finanzierungsstandbein neben den Beitragseinnahmen ist der als Ausfallshaftung konzipierte „Bundesbeitrag“. In den gesetzlichen Regelungen zur Pensionsversicherung ist festgelegt, dass die Differenz zwischen den Beitragseinnahmen und den Ausgaben aus Bundesmit-

17 In Österreich wird der Jahreslohn in aller Regel in 14 Monatsbeträgen ausbezahlt. Der 13. und der 14. Bezug (Urlaubs- und Weihnachtsgeld) sind ebenfalls beitragspflichtig. 
teln zu begleichen ist. Über die Ausfallshaftung des Bundes werden aktuell etwa $18 \%$ des Pensionsaufwands in der gesetzlichen Pensionsversicherung finanziert. Der Anteil schwankt von Jahr zu Jahr, abhängig von der Entwicklung einerseits der Beitragseinnahmen und andrerseits der Pensionskosten.

Wie die ausgewiesenen Werte zeigen, liegt der Bundesbeitrag derzeit auf einem vergleichsweise niedrigen Niveau, gemessen sowohl in Prozent der Pensionsausgaben als auch als BIP-Anteil. Hier spiegeln sich einerseits die gute Entwicklung der Wirtschaft und der Beschäftigtenzahlen in den letzten Jahren und andrerseits die Effekte der durchgeführten Pensionsreformen.

$\mathrm{Zu}$ beachten ist, dass sich die in Tabelle 3 ausgewiesenen Werte auf die gesamte gesetzliche Pensionsversicherung beziehen. Bei getrennter Betrachtung der Versicherungsträger für Unselbständige und Selbständige zeigen sich erhebliche Unterschiede: 2018 deckte die Ausfallshaftung des Bundes bei den Unselbständigen $12 \%$ und bei den Selbständigen hingegen $52 \%$ des Pensionsaufwands ${ }^{18}$ - ein Ungleichgewicht, das von Gewerkschaftsseite immer wieder kritisiert wird.

\subsection{Betriebspensionen}

Betriebspensionen haben in Österreich traditionell eine relativ geringe Bedeutung. Nur in ganz wenigen Ausnahmefällen (z.B. Universitäten) gibt es branchenweite Regelungen. Nach Berechnungen des Österreichischen Instituts für Wirtschaftsforschung haben gut $30 \%$ der Arbeitnehmer eine Anwartschaft auf eine Betriebspension (Url 2012). In vielen Fällen werden allerdings nur sehr niedrige Beiträge bezahlt, wie z.B. 0,75\% Arbeitgeberbeitrag im 2008 gestarteten Betriebspensionssystem für Beschäftigte im öffentlichen Dienst.

Ähnlich wie in Deutschland gibt es verschiedene Durchführungswege. Am weitesten verbreitet sind Zusagen über „Pensionskassen“, die ähnlich gestaltet sind wie die deutschen Pensionsfonds. Eigentümer dieser Kassen sind im Regelfall Versicherungen und/oder Banken. Mitbestimmungsregelungen sehen vor, dass die Anwartschafts- und Leistungsberechtigten Vertreter in den Aufsichtsrat entsenden können, die Mehrheit liegt allerdings bei den Kapitalvertretern.

18 Hauptverband der österreichischen Sozialversicherungsträger (2019e). 
Im Betriebspensionsgesetz ist u.a. normiert, dass mindestens $50 \%$ der Beitragsleistung zu einem Betriebspensionssystem vom Arbeitgeber bezahlt werden müssen. Für Betriebe mit Betriebsrat ist vorgeschrieben, dass der Beitritt zu einem ausgelagerten Versorgungssystem - wie z.B. zu einer Pensionskasse - nur zulässig ist, wenn dazu vorgelagert eine Betriebsvereinbarung abgeschlossen wurde. ${ }^{19}$

\subsection{Ausblick}

Modellrechnungen der OECD zeigen, dass die österreichische Pensionsversicherung auch den heute Jüngeren einen relativ guten Einkommensersatz bietet. So zeigt z.B. die Standardberechnung 78 \% Brutto- bzw. 92 \% NettoEinkommensersatz. Angenommen werden dabei ein Berufseinstieg zum Alter 20 und durchgehende Erwerbstätigkeit mit Durchschnittseinkommen bis zum Regelpensionsalter (OECD 2017, S. 103). Wenngleich zu bedenken ist, dass die real erzielbaren Einkommensersatzraten in aller Regel um einiges niedriger liegen werden (kürzere Versicherungsdauer, Berufsunterbrechungen, Niedrigverdienst-/Teilzeitphasen, etc.) signalisieren diese Werte ein Versorgungsniveau im Alter, das nur sehr wenige Pensionssysteme anderer Länder erreichen.

Wie in jedem Pensionssystem, in dem Dauer und Höhe der Beitragszahlung bzw. des versicherten Einkommens zentrale Determinanten der Leistungsbemessung sind, ist die konkrete Leistungshöhe in hohem Maß vom vorgelagerten Erwerbsverlauf abhängig. Die Sicherstellung guter Pensionen auch für die heute Jüngeren erfordert damit fürs erste, dass ihnen die Möglichkeit einer guten Erwerbsintegration mit entsprechender Entlohnung geboten wird - ein Aspekt, der in der Diskussion über die Perspektiven der Alterssicherung oft zu kurz kommt und nicht selten sogar zur Gänze ausgeblendet wird. Absehbar ist, dass soziale Ausgleichsmechanismen, wie die Anrechnung von Kindererziehungszeiten oder Zeiten der Arbeitslosigkeit auch in Zukunft nicht ausreichen werden, um negative Effekte langer Unterbrechungen, prekärer Arbeitsformen, etc. in der Pension auszugleichen.

In der öffentlichen Debatte über die Zukunft der Pensionen steht in aller Regel die Entwicklung der Ausgaben im Mittelpunkt. Unter Verweis auf den stark steigenden Anteil der Älteren an der Gesamtbevölkerung wird von wirtschaftsnahen Experten - ähnlich wie in anderen Ländern -

19 Näheres zu den Betriebspensionen in Österreich siehe Wöss (2018). 
immer wieder die Gefahr „explodierender“ Pensionskosten in den Raum gestellt. In den vorliegenden Langzeit-Rechnungen finden diese Befürchtungen keine Deckung.

Tabelle 4: Vorausberechnung der öffentlichen Pensionskosten und des Anteils älterer Menschen an der Gesamtbevölkerung 2020-2070

\begin{tabular}{ccc}
\hline & $\begin{array}{c}\text { Öffentliche Pensions- } \\
\text { ausgaben in \% des BIP }\end{array}$ & $\begin{array}{c}\text { Anteil der Altersgruppe 65+ } \\
\text { an der Gesamtbevölkerung }\end{array}$ \\
\hline 2020 & $13,9 \%$ & $19,0 \%$ \\
2030 & $14,4 \%$ & $22,6 \%$ \\
2040 & $14,9 \%$ & $25,5 \%$ \\
2050 & $14,6 \%$ & $27,0 \%$ \\
2060 & $14,7 \%$ & $29,2 \%$ \\
2070 & $14,3 \%$ & $30,4 \%$ \\
\hline
\end{tabular}

Quelle: EU Ageing Report 2018, Annex Part IV (Austria)

Trotz massiver Alterung der Bevölkerung wird nur ein relativ geringer Anstieg bei den öffentlichen Pensionsausgaben erwartet. Dahinter steht u.a. die Einschätzung, dass die durchgeführten Pensionsreformen vor allem in mittel- bis längerfristiger Perspektive zu beträchtlichen Einsparungen führen werden (höheres durchschnittliches Pensionseintrittsalter, etwas niedrigere Einkommensersatzraten, etc.). Dazu kommt, dass bei den Ausgaben für Beamtenpensionen und für Hinterbliebenenpensionen mit einem beträchtlichen Rückgang der Kosten gerechnet wird.

\section{Vergleich Österreich - Deutschland}

Wie die deutsche Gesetzliche Rentenversicherung folgt auch die österreichische Pensionsversicherung dem Bismarck'schen Modell. Im Zentrum steht in beiden Ländern ein im Umlageverfahren finanziertes gesetzliches Versicherungssystem mit einer engen Verbindung zwischen Beiträgen und Leistungen. Bei genauerem Hinsehen zeigen sich allerdings ganz erhebliche Unterschiede, insbesondere:

- Die österreichische Pensionsversicherung bietet ein wesentlich höheres Leistungsniveau - bei den heute Jüngeren fällt Deutschland noch weiter zurück. 
- In Österreich sind (fast) alle Erwerbstätigen in die gesetzlichen Pensionssysteme eingebunden, der Versichertenkreis ist damit wesentlich breiter als in Deutschland.

- Die Mindestsicherung im Alter ist in Österreich in die gesetzliche Pensionsversicherung integriert, die Leistungen sind höher und es erfolgt keine Vermögensprüfung.

- Österreich wendet einen wesentlich höheren BIP-Anteil für die Finanzierung der gesetzlichen Pensionen auf.

Teils haben diese Unterschiede eine lange Vorgeschichte, teils sind sie Ergebnis der sehr unterschiedlichen Reformen zu Beginn der 2000er Jahre, die im Ergebnis zu einer sehr unterschiedlichen Ausrichtung der Systeme geführt haben. In Deutschland wurde damals das Ziel der Lebensstandardsicherung durch die gesetzliche Rente aufgegeben und ein erheblicher Teil der Verantwortung für die Alterssicherung auf Betriebs- und Privatrenten verlagert. In Österreich gab es sehr ähnliche Reformbestrebungen, allerdings scheiterten diese am breitflächigen Widerstand. Das Ziel der weitgehenden Lebensstandardsicherung durch eine Pension aus dem gesetzlichen System blieb trotz umfassender Änderungen aufrecht.

Die Ausweitung des Versichertenkreises über die unselbständig Erwerbstätigen hinaus begann bereits in den 1950er Jahren. ${ }^{20}$ Der letzte wesentliche Schritt erfolgte 1996/1997 mit der Pflichtversicherung auch von neuen Erwerbsformen im Grenzbereich zwischen Unselbständigkeit und Selbständigkeit.

Ein weiterer wesentlicher Unterschied besteht darin, dass in Österreich auch die Beamtenversorgung in den Reformprozess eingebunden wurde. Langfristig werden dort sowohl das Beitrags- als auch das Leistungsrecht an die Regelungen der gesetzlichen Pensionsversicherung angeglichen.

Der Beitragssatz zur gesetzlichen Pensionsversicherung beträgt in Österreich 22,8\% und liegt damit deutlich höher als der entsprechende Wert in Deutschland mit derzeit 18,6\%. Wird allerdings der in Deutschland vorgesehene 4-\%-Beitrag zur privaten Riester-Rente mit ins Kalkül genommen, gleicht sich dieser Unterschied weitgehend aus. Der Arbeitnehmer- bzw. Eigenbeitrag liegt bei Gesamtbetrachtung in Deutschland sogar um einiges höher als in Österreich.

Die Gegenüberstellung der jeweiligen Rentenzahlbeträge macht die massiven Unterschiede beim Leistungsniveau der gesetzlichen Renten deutlich (Tabelle 5):

201958 wurden die Gewerbetreibenden in die Pflichtversicherung der gesetzlichen Pensionsversicherung eingebunden. 
Tabelle 5: Durchschnittliche Brutto-Rentenzablbeträge bei neu zuerkannten Altersrenten/Alterspensionen 2016 (Unselbständige/inkl. zwischenstaatliche Teilleistungen)

\begin{tabular}{lcc}
\hline & Deutschland & Österreich \\
\hline Männer & $€ 1.120$ & $€ 2.001^{*}$ \\
Frauen & $€ 757$ & $€ 1.253^{*}$ \\
\hline
\end{tabular}

Quelle: Blank et al. (2018)

"Jahreszwölftel (zum einfacheren Vergleich mit Deutschland)

$\mathrm{Zu}$ beachten ist, dass die konkreten Rentenzahlbeträge nicht nur durch die jeweiligen Berechnungsformeln bestimmt werden, sondern auch durch andere Faktoren: So sind in Österreich 15, in Deutschland hingegen nur 5 Versicherungsjahre für die Zuerkennung einer Alterspension bzw. -rente erforderlich. Ein ganz wesentlicher Faktor ist darüber hinaus in beiden Ländern der vorgelagerte Erwerbsverlauf. Am besten vergleichbar sind aus diesen Gründen die Rentenzahlbeträge bei den Männern. Hier spielt die Vorgabe von 15 bzw. fünf Versicherungsjahren praktisch nur eine sehr geringe Rolle und auch die Karriereverläufe sind ähnlich.

Tabelle 6: Jährliche Rentengutschrift in \% des versicherten Erwerbseinkommens

\begin{tabular}{ccc}
\hline \multicolumn{2}{c}{ Deutschland } & Österreich \\
Rentenantritt 2016 & Berufseinstieg 2016 & Pensionskonto-Recht \\
\hline $0,99 \%$ & $0,83 \%$ & $1,74 \%{ }^{21}$ \\
\hline
\end{tabular}

Quelle: Türk E./Blank F. (2017)

Anmerkung: Die für Deutschland ausgewiesenen Werte ergeben sich durch Gegenüberstellung des aktuellen bzw. vorausberechneten Werts eines Entgeltpunkts in Relation zur Höhe des versicherten Durchschnittseinkommens. Der für Österreich angegebene Wert gilt für Pensionsanwartschaften, die ab 2005 erworben wurden. Ältere Anwartschaften wurden im Regelfall höher bewertet.

21 Die durchschnittliche effektive Pensionsgutschrift liegt mit 1,74\% etwas niedriger als der Pensionskonto-Prozentsatz (1,78\%), weil im Jahr des Pensionsantritts keine Aufwertung des Kontoguthabens mehr erfolgt. 
Eine vergleichende Betrachtung unter Ausblendung von Faktoren wie Anspruchsvoraussetzungen, Erwerbskarrieren, Pensionseintrittsalter, etc. erlaubt die Gegenüberstellung der jeweiligen Renten- bzw. Pensionsgutschriften pro Versicherungsjahr.

Tabelle 6 bestätigt, was bereits in der Gegenüberstellung der Rentenzahlbeträge deutlich wurde: Die österreichische Pensionsversicherung bietet ein wesentlich höheres Leistungsniveau als die deutsche Rentenversicherung. Für heutige Berufseinsteiger ist die jährliche Rentengutschrift in Österreich sogar mehr als doppelt so hoch wie in Deutschland! Sehr augenscheinlich wird das sehr unterschiedliche Leistungsniveau auch beim Vergleich der erforderlichen Versicherungsjahre zur Erreichung eines bestimmten Renten- bzw. Pensionsniveaus. Türk und Blank (2017) haben untersucht, wie viele Beitragsjahre im jeweiligen System erforderlich sind, um eine Rente/Pension in Höhe der Armutsgefährdungsgrenze zu erreichen. Auch in dieser Betrachtungsweise zeigen sich gravierende Unterschiede.

Tabelle 7: Für eine Regelaltersrente in Höhe der Armutsgefährdungsgrenze sind ..... Beitragsjabre erforderlich

\begin{tabular}{|c|c|c|c|}
\hline \multirow[b]{2}{*}{ Verdienst } & \multicolumn{2}{|c|}{ Deutschland } & \multirow{2}{*}{$\begin{array}{c}\text { Österreich } \\
\text { Pensionskonto- } \\
\text { recht }\end{array}$} \\
\hline & $\begin{array}{c}\text { Rentenantritt } \\
2016\end{array}$ & $\begin{array}{c}\text { Berufseinstieg } \\
2016\end{array}$ & \\
\hline $\begin{array}{c}100 \% \text { des Durch- } \\
\text { schnittseinkommens }\end{array}$ & 40,6 Jahre & 48,0 Jahre & 26,0 Jahre \\
\hline $\begin{array}{c}75 \% \text { des Durch- } \\
\text { schnittseinkommens }\end{array}$ & 54,1 Jahre & 64,1 Jahre & 34,7 Jahre \\
\hline
\end{tabular}

Wie diese Werte zeigen, muss in Deutschland damit gerechnet werden, dass in Zukunft selbst Durchschnittsverdiener nur mehr in Ausnahmefällen eine gesetzliche Rente in Höhe der Armutsgefährdungsgrenze erreichen werden. Liegt das Erwerbseinkommen z.B. nur bei $75 \%$ des Durchschnitts, ist das in aller Regel bereits heute unmöglich! In Österreich sind demgegenüber bei Durchschnittsverdienst 26 und bei einem Verdienst in Höhe von 75 \% des Durchschnitts 34,7 Versicherungsjahre für eine Pension in Höhe der Armutsgefährdungsgrenze erforderlich. Wenig überraschend ist in Anbetracht derartiger Werte, dass die Armutsgefährdungsquoten im Alter tendenziell in Deutschland nach oben und in Österreich nach unten weisen (Abbildung 1). 
Abbildung 1: Armutsgefährdungsquoten in der Altersgruppe 65+ Deutschland - Österreich im Vergleich 2007-2017, Prozent

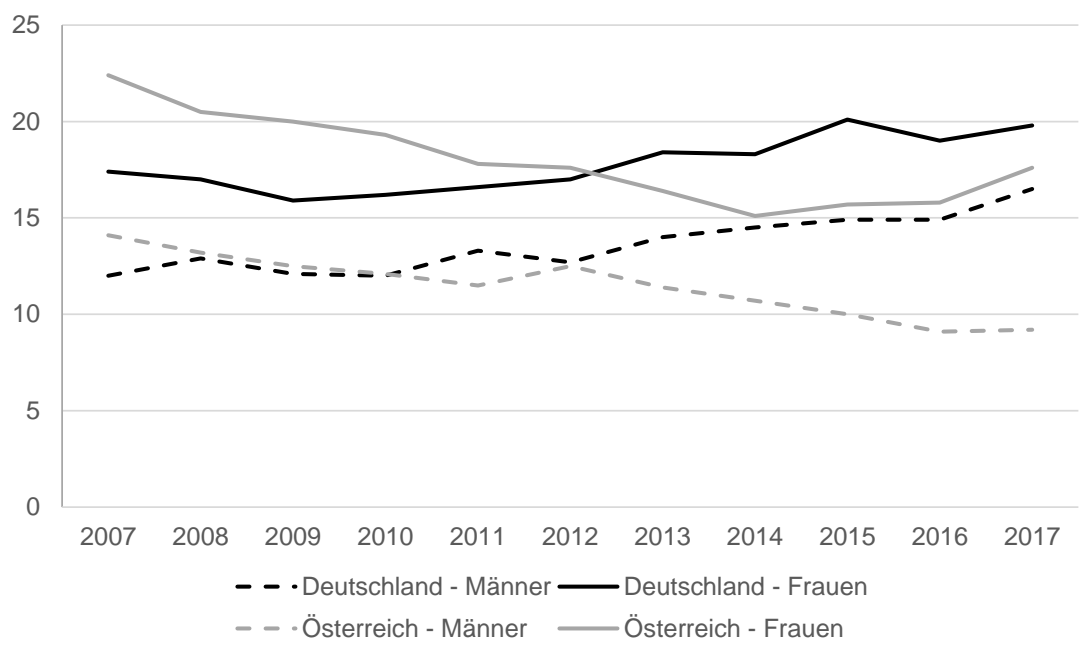

Quelle: Türk/Blank (2017), aktualisiert; Datenbasis Eurostat 2020

\section{Die Schlüsselrolle des Arbeitsmarktes in der Alterssicherung}

Im Zentrum der Diskussion über die Zukunft der Alterssicherungssysteme steht seit mehreren Jahrzehnten der demografische Wandel bzw. die erwartete kräftige Alterung der Bevölkerung. Sorgen bereitet dabei vor allem die künftige Entwicklung der Zahl der Rentner/Pensionisten in Relation zur Zahl der Beitragszahler. Das gilt für Österreich wie für Deutschland.

Blicken wir zuerst auf die Vorhersagen der Demografen bzw. auf die erwartete Entwicklung der Altenquote, wie sie den Langzeitrechnungen im EU Ageing Report 2018 zugrunde gelegt ist. Demnach wird die „old-age dependency ratio“, definiert als Zahl der Menschen in der Altersgruppe ab 65 relativ zur Altersgruppe 15 bis 64, zwischen 2016 und 2070 in Deutschland von 32,2 \% auf 55,9\% und in Österreich von $27,6 \%$ auf $54,4 \%$ steigen. Für die EU insgesamt (einschließlich Großbritannien) wird ein Anstieg von $29,6 \%$ auf $51,2 \%$ erwartet. $^{22}$

22 EU-Kommission (2018, Table III.1.60). 
Evident ist, dass demografische Verschiebungen in diesem Ausmaß enorme Herausforderungen für unsere Gesellschaften als Ganzes und insbesondere für die Alterssicherungssysteme mit sich bringen. Umso bedauerlicher ist es, dass der Blick auf zentrale Handlungsoptionen immer wieder verstellt wird durch grobe Fehldarstellungen, in denen die Zahl der Beitragszahler mit der Zahl der Menschen im Erwerbsalter und die Zahl der Rentner mit der Zahl der Menschen über einer bestimmten Altersgrenze gleichgesetzt wird. So verlautbarte z.B. die EU-Kommission bei der Vorstellung des Grünbuchs zu den Renten im Jahr 2010: „Erwartet wird, dass sich die Zahl der Rentner in Europa bis 2060 relativ zur Zahl der Beitragszahler verdoppeln wird - diese Situation ist unhaltbar". Ähnlich die EU-Kommission bei der Vorstellung des EU Ageing Reports 2015, wo in einer europaweit verbreiteten Grafik eine bevorstehende Verschiebung der Relation zwischen Erwerbstätigen und Rentnern von heute 4:1 auf 2:1 suggeriert wurde.

Allerdings gibt es keine einzige seriöse Datenquelle, die solche Behauptungen belegen könnte. Sowohl der Ausgangswert als auch die behauptete Verschlechterung der Relation zwischen Rentnern und Beitragszahlern sind evident falsch. Die EU-Kommission selbst hat in ihrem Ageing Report 2015 für den EU-Raum die Zahl der Erwerbstätigen mit 214 Mio. und die Zahl der Rentner bzw. Pensionisten mit 124 Mio. ausgewiesen (EU-Kommission 2015), d.h. dass die tatsächliche Relation zwischen Erwerbstätigen und Rentnern war nicht 4:1 ist, sondern gerade mal 1,7:1.

Wie kommt es zu den zitierten Fehlbehauptungen? Die Erklärung ist verblüffend einfach: Die Relation zwischen verschiedenen Altersgruppen wird ohne Umschweife in die Relation zwischen Erwerbstätigen und Rentnern umgedeutet! Problematisch ist das nicht nur, weil es fernab der Realität ist und ein völlig falsches Bild vermittelt. Mindestens ebenso problematisch ist, dass mit der unrichtigen Gleichsetzung der Zahl der Menschen im Erwerbsalter mit der Zahl der Erwerbstätigen der Blick auf eine zentrale Handlungsoption im Umgang mit der demografischen Alterung verstellt wird, der besseren Erwerbsintegration der Menschen im Erwerbsalter quer durch alle Altersgruppen.

Der enorme Unterschied zwischen demografischer „Altenquote“ und wirtschaftlicher „Abhängigkeitsquote“ hat vor allem zwei Gründe: Erstens ist etwa ein Drittel der Menschen im Erwerbsalter nicht erwerbstätig - teils weil sie in Ausbildung sind (Schüler, Studierende) sind, mehr noch aber wegen Arbeitslosigkeit, schlechter Vereinbarkeit Beruf/Familie, Invalidisierung, Frühpension, etc. Zweitens sind etwa ein Viertel der Rentner jünger als 65 (EU-Raum / EU- Kommission 2015). 
Ökonomisch betrachtet macht vor diesem Hintergrund die Gegenüberstellung der Zahl der Menschen im Erwerbsalter mit der Zahl der Menschen im höheren Alter wenig Sinn. Klar zum Ausdruck gebracht wird das im EU Weißbuch zu den Pensionen: „Die Alterungsproblematik wird häufig anhand der Verdoppelung der Alterslastquote (Verhältnis der Bevölkerung 65+ zur Bevölkerung 15-64) von $26 \%$ im Jahr 2010 auf $50 \%$ im Jahr 2050 aufgezeigt. Der springende Punkt ist jedoch die wirtschaftliche Abhängigkeitsrate, die wie folgt definiert ist: Arbeitslose und Personen im Ruhestand als Prozentsatz der Erwerbstätigen. “23 Unverständlich ist, warum eine so definierte „wirtschaftliche Abhängigkeitsrate“ in den Arbeiten der EU-Kommission, z.B. in den Szenarien-Rechnungen im Ageing Report, bisher keine Berücksichtigung gefunden hat.

Wie hoch das Potential einer Strategie der „besseren Erwerbsintegration in allen Altersgruppen“ ist, um den demografisch bedingten Anstieg der wirtschaftlichen Abhängigkeitsrate einzudämmen, wurde zuletzt in Szenarien-Rechnungen zu Deutschland aufgezeigt (Türk et al. 2018). Berechnet wurde, wie sich die Entwicklung der Relation zwischen Arbeitslosen und Rentnern auf der einen und Erwerbstätigen auf der anderen Seite entwickeln würde, wenn Deutschland bis zum Jahr 2050 Erwerbsquoten erreichen würde, wie sie derzeit in Schweden gegeben sind.

23 EU-Kommission (2012), Abschnitt 2.3. 


\section{Abbildung 2: Altenquote/ökonomische Abhängigkeitsquote - Deutschland} 2013-2060. Änderung in Prozent (Ausgangswert $2013=100$ )

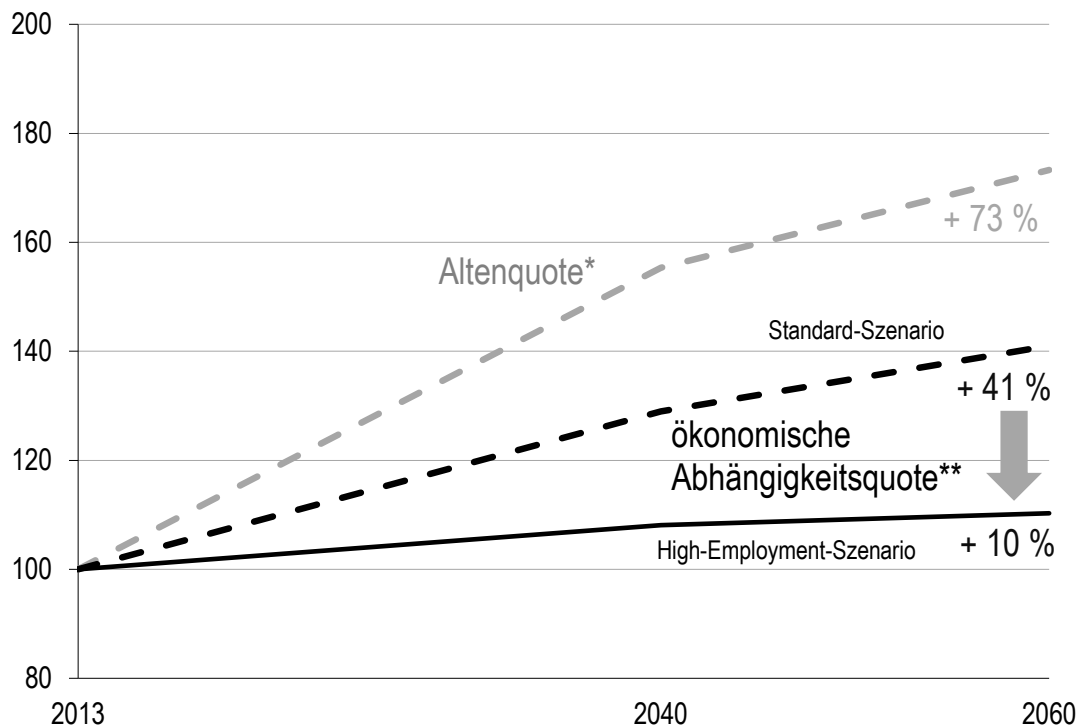

Quelle: Türk et al. 2018; Grafik: AK Wien

Anmerkungen: *Verhältnis Personen im Renten- zu Personen im Erwerbsalter (ab 65-jährige/15-64); ${ }^{* *}$ Verhältnis (Rentner + Arbeitslose) / Erwerbstätige (ohne Mini-Jobber)

Die der Abbildung 2 zugrundeliegenden Berechnungen zeigen:

a) Der erwartete Anstieg der ökonomischen Abhängigkeitsquote wird aller Voraussicht nach wesentlich weniger stark ausfallen als der Anstieg der Altenquote mit $+73 \%$.

b) Würde es bis 2060 gelingen, die derzeit in Schweden gegebenen Erwerbsquoten und eine deutliche Reduktion der Arbeitslosigkeit zu erreichen, würde die Zahl der Arbeitslosen und Rentner in Relation zur Zahl der Erwerbstätigen nur um 10\% steigen - und nicht wie aktuell angenommen um $41 \%$.

Deutlich gemacht wird mit dieser Szenarien-Rechnung, dass die Relation zwischen Leistungsbeziehern und Beitragszahlern (bzw. Erwerbstätigen) bei weitem nicht nur - wie oft behauptet - von der Entwicklung der Altenquote und vom gesetzlichen Renten- bzw. Pensionsalter abhängt. Eine ganz zentrale Rolle spielt dabei auch der Arbeitsmarkt bzw. wie gut/ 
schlecht die Menschen im Erwerbsalter in das Erwerbsleben eingebunden sind. Klar ist, dass von einer guten Einbindung in das Erwerbsleben nur bei guter Qualität der Arbeit und der Arbeitseinkommen gesprochen werden kann.

\section{Programm der neuen Regierung (Koalition ÖVP/Grüne)}

$\mathrm{Zu}$ Jahresbeginn 2020 wurde in Österreich eine neue Regierung angelobt. Für viele überraschend verständigten sich die konservative ÖVP und die eher als politisch linksstehend eingeschätzten - Grünen auf eine Regierungskoalition. Was ist im Bereich der Alterssicherungspolitik von dieser Regierung zu erwarten? Im Regierungsprogramm wird der Abschnitt Pensionen mit folgenden Sätzen eingeleitet: „Das österreichische Pensionssystem zeichnet sich durch Sicherheit und Klarheit aus. Es gibt zwar immer wieder Adaptionsbedarfe, aber wir brauchen keine grundsätzliche Neuausrichtung. Was es braucht, sind Bemühungen, Lücken und Ungerechtigkeiten zu schließen und Nachhaltigkeit sicherzustellen." Die konkret angeführten Reformvorhaben zur Beseitigung von „Lücken und Ungerechtigkeiten" 24 und zur Sicherstellung von „Nachhaltigkeit" sind überwiegend auf den Arbeitsmarkt ausgerichtet. Angesprochen ist in diesem Zusammenhang auch das Ziel, das „effektive Pensionsantrittsalter deutlich zu erhöhen".

Das Programm lässt erwarten, dass es in den kommenden Jahren zu keinen großen Änderungen im Pensionsrecht kommen wird. Von einer weiteren "großen Pensionsreform", wie das wirtschaftsnahe Pensionsexperten immer wieder mal öffentlichkeitswirksam einfordern, ist keine Rede. Die Fokussierung auf den Arbeitsmarkt (bessere Vereinbarkeit Beruf/Familie, Gesundheitsschutz, altersgerechte Arbeitsgestaltung, Rehab-Maßnahmen, etc.) als Instrument auch der Pensionspolitik könnte sogar als Schulterschluss mit den Gewerkschaften gedeutet werden. Auch die Zielsetzung „das effektive Pensionsantrittsalter deutlich zu erhöhen“ entspricht im Grundsatz - bei Sicherstellung entsprechender Erwerbschancen - der Positionierung der Gewerkschaften.

Konfliktstoff mit den Gewerkschaften bietet allerdings ein Punkt, der im Regierungsprogramm nicht angesprochen ist: die im Herbst 2019 ohne Vorbegutachtung im Parlament beschlossene Streichung der Pensionsab-

24 Konkret angesprochen sind die vielen niedrigen Frauenpensionen und die Altersarmut. 
schläge bei Vorliegen von 45 Pflichtversicherungs- bzw. Erwerbsjahren (siehe oben, Fußnote 14). Die Regierungsspitze hat wenige Tage nach Vorlage ihres Programms eine Überarbeitung dieser von den Gewerkschaften begrüßten Regelung angekündigt. Allerdings wurde weder zum Inhalt der geplanten Änderung noch zum Zeitplan Genaueres verlautbart.

Mit Interesse erwartet wird auch, welche konkreten Maßnahmen der von den Grünen gestellte Sozialminister zur angekündigten Verbesserung der niedrigen Frauenpensionen und - damit verbunden - zur Reduktion der Altersarmut vorschlagen wird. Konkret angekündigt wurde bisher nur die Einführung eines automatischen Splittings der Pensionsanwartschaften von Eltern bis zum 10. Lebensjahr der Kinder, verbunden mit der Möglichkeit zu einem Opting-out.

Ziemlich sicher ist jedenfalls, dass auch die Regierungskoalition ÖVPGrüne an den - im Vergleich zu Deutschland - sehr starken gesetzlichen Renten festhalten wird. Österreich wird damit auch in Zukunft als Referenzland dienen können für alle, die aus guten Gründen in Deutschland eine Stärkung der gesetzlichen Rente fordern. Gelingt es dem grünen Sozialminister, wirksame Maßnahmen zur Verbesserung der Frauenpensionen auf den Weg zu bringen, könnte das Interesse am „österreichische Modell“ sogar weiter steigen.

\section{Literatur}

Blank F./Logeay C./Türk E./Wöss J./Zwiener R. (2018): Ist das österreichische Pensionssystem nachhaltig?, in: Wirtschaftsdienst 98 (3), S. 193-199

Blank F./Logeay C./Türk E./Wöss J./Zwiener R. (2016): Alterssicherung in Deutschland und Österreich: Vom Nachbarn lernen? WSI-Report Nr. 27, 1/2016, Düsseldorf

Bundeskanzleramt (2019): Nationales Reformprogramm, Österreich, Wien

Bundesministerium für Arbeit, Soziales, Gesundheit und Konsumentenschutz (2018): Sozialstaat Österreich. Leistungen, Ausgaben und Finanzierung 2018, Wien

EU-Kommission (2012): An Agenda for Adequate, Safe and Sustainable Pensions. White Paper, Brüssel

EU-Kommission (2015): The 2015 Ageing Report, Economic and budgetary projections for the 28 EU Member States (2013-2060), Brüssel

EU-Kommission (2018): The 2018 Ageing Report. Economic \& Budgetary Projections for the 28 EU Member States (2016-2070), Luxemburg 
Eurostat (2020): Armutsgefährdungsquote älterer Personen nach Geschlecht und nach ausgewählten Altersgruppen - EU-SILC und ECHP Erhebungen, Tabelle Code ilc_pnp1, https://ec.europa.eu/eurostat/de/data/database (abgerufen am 17.02.2020)

Hauptverband der österreichischen Sozialversicherung (2011): Statistische Daten aus der Sozialversicherung. Pensionsversicherung. Berichtsjahr 2010, Wien

Hauptverband der österreichischen Sozialversicherung (2019a): Statistisches Handbuch der österreichischen Sozialversicherung 2019, Wien

Hauptverband der österreichischen Sozialversicherung (2019b): Statistische Daten aus der Sozialversicherung. Pensionsversicherung. Berichtsjahr 2018, Wien

Hauptverband der österreichischen Sozialversicherung (2019c): Statistische Daten aus der Sozialversicherung, Verteilung des monatlichen Pensionseinkommens. Neuzuerkennungen 2018, Wien

Hauptverband der österreichischen Sozialversicherung (2019d): Statistische Daten aus der Sozialversicherung. Verteilung des monatlichen Pensionseinkommens. Pensionsstände Dezember 2018. Berichtsjahr 2018, Wien

Hauptverband der österreichischen Sozialversicherungsträger (2019e): Jahresbericht der österreichischen Sozialversicherung 2019, Wien

OECD (2017): Pensions at a Glance 2017. OECD and G20 Indicators, Paris

Türk E. (2019): Die Entwicklung des effektiven Pensionsantrittsalters, in: Wirtschaft und Gesellschaft 45 (3), S 367-389

Türk E./Blank F. (2017): Niedrigrenten, Mindestsicherung und Armutsgefährdung Älterer. Ein Vergleich zwischen Österreich und Deutschland (Teil 1), in: Soziale Sicherheit 70 (7-8/2017), S. 460-461

Türk E./Blank F. (2017): Armutsgefährdung Älterer - Deutschland und Österreich im Vergleich. A\&W Blog 30.11.2017, https://awblog.at/armutsgefaehrdung-aelterer-deutschland-und-oesterreich-im-vergleich/ (abgerufen am 21.03.2020)

Türk E./Blank F./Logeay C./Wöss J./Zwiener R. (2018): Den demografischen Wandel bewältigen: Die Schlüsselrolle des Arbeitsmarktes, IMK Report 137, April 2018, Düsseldorf

Wöss, J. (2018): Austria: Occupational Welfare at the edge of statutory social insurance, in: Natali D./Pavolini E./Vanhercke B. (Hrsg.): Occupational Welfare in Europe, Brüssel, S. 147-171 
